Int. J. Dev. Biol. 50: 463-471 (2006)

doi: $10.1387 / \mathrm{ijdb} .052106 \mathrm{ym}$

Original Article

\title{
Two modes of action by which Xenopus hairy2b establishes tissue demarcation in the Spemann-Mangold organizer
}

\author{
YASUHITO MURATO ${ }^{1,2}$, MAMI YAMAGUTI ${ }^{1,2}$, MIHO KATAMURA ${ }^{3}$, KEN W.Y. CHO ${ }^{4}$ \\ and CHIKARA HASHIMOTO*,1,2

\begin{abstract}
${ }^{1}$ Department of Biology, Graduate School of Science, Osaka University, Toyonaka, Osaka, Japan, 2JT Biohistory Research Hall, Takatsuki, Osaka, Japan, ${ }^{3}$ Graduate School of Medical Science, Kyoto University, Sakyoku, Kyoto, Japan and ${ }^{4}$ Department of Developmental and Cell Biology and the Developmental Biology Center, University of California, Irvine, USA
\end{abstract}

\begin{abstract}
The Hairy and Enhancer-of-Split (HES) family of transcriptional repressors plays important roles in pattern formation during development throughout the animal kingdom. Generally, HES proteins repress the expression of genes specific for neighboring tissues to maintain the nature of cells expressing HES proteins, resulting in pattern formation. Xhairy2b, a Xenopus HES, establishes the prospective anterior prechordal mesoderm identity in the Spemann-Mangold organizer by both inducing specific genes and repressing the genes specific for neighboring tissues. Here we report that $X$ hairy $2 b$ has two modes of action, each of which corresponds to inductive and repressive functions. We show that the inductive function is independent of direct transcriptional regulation and is exhibited by the C-terminal WRPW tetrapeptide motif alone, although it induces the expression of a wide variety of the organizer genes that Xhairy2b represses. The transcriptional repression by $X$ hairy $2 b$ is responsible for only the repressive function. We propose that the activity of the WRPW motif intrinsically induces the expression of genes specific for the organizer in a rather non-specific manner to ensure the organizer environment. Then, the transcriptional repression selectively down-regulates the expression of some of these genes, resulting in the regionalization of the axial mesoderm. Our study provides new insight into how a region of the vertebrate embryo is demarcated by one dual-functional transcription factor in the early stages of development.
\end{abstract}

KEY WORDS: Xhairy2b, Organizer, WRPW motif, pattern formation, Xenopus

\section{Introduction}

In the early development of vertebrate as well as invertebrate organisms, transcription factors play crucial roles as key switches that dramatically change or firmly sustain the nature of cells. The transcription factors exert their effects on transcription by directly binding to a specific target sequence. Naturally, deletion of the DNA binding domain will severely attenuate their ability to regulate transcription. However, some transcription factors are able to regulate gene expression through different mechanisms. One well-known example is Drosophila Fushi-tarazu (FTZ; Kuroiwa et al., 1984), which is able to regulate gene expression even if the homeobox is deleted (Copeland et al., 1996). It was eventually shown that FTZ physically interacts with the nuclear orphan receptor FTZ-F1 as a coactivator (Suzuki etal., 2001). Clearly, the example of FTZ and FTZ-F1 suggests that some transcription factors have acquired functions as non-transcription factors, especially through protein-protein interaction.
The HES (hairy and Enhancer-of-Split) family basic helix-loophelix (bHLH) transcription factors are involved in transcriptional regulation for pattern formation and regulation of growth and differentiation in the early development of both vertebrate and invertebrate organisms (Fisher and Caudy, 1998; Davis and Turner, 2001). The HES proteins, similar to other bHLH proteins, form dimers (both homo- and heterodimers) via the HLH domain and bind to the target sequence (i.e., N-box) through the basic arms (Fisher and Caudy, 1998; Davis and Turner, 2001). In addition to transcriptional regulation through direct DNA binding, previous studies have shown that certain vertebrate as well as fly HES proteins function as inhibitors of bHLH activators (Bae et al., 2000; Giagtzoglou et al., 2003). Collectively, the HES proteins seem to function not only as transcription factors but also as non-

Abbreviations used in this paper: bHLH, basic helix-loop-helix; EnR, Engrailed repression domain; FTZ, Fushi-tarazu; HES, hairy and enhancer-of-split transcription factor. 
transcription factors possibly by protein-protein interaction, suggesting functional flexibility and complexity depending on the developmental context.

Xhairy2b, a Xenopus HES, is expressed in the deep layer of the dorsal lip, the Spemann-Mangold organizer (Sander and Faessler, 2001), and plays important roles in tissue demarcation (Tsuji et al., 2003; Yamaguti et al., 2005). At the onset of gastrulation, Xhairy $2 b$ represses the expression of genes specific for the ventral mesoderm and the anterior endoderm, resulting in the maintenance of the dorsal mesoderm identity for trunk formation (Yamaguti et al., 2005). In late gastrulae, Xhairy2b expression becomes predominant in the anterior prechordal mesoderm and loss-of-function experiments showed that Xhairy2b is required for repressing the expression of genes specific for the posterior prechordal mesoderm and the chordamesoderm (Yamaguti etal.,
2005). These observations clearly show that Xhairy2b as a transcriptional repressor maintains the identity of tissue where Xhairy $2 b$ itself is expressed by repressing the expression of genes specific for neighboring tissues. Our previous study (Yamaguti et al., 2005) also showed that Xhairy2b has another important biological function in early gastrulae, which is the ability to induce secondary body axis and organizer-specific gene expression, such as admp (Moos etal., 1995) and follistatin(HemmatiBrivanlou et al., 1994; lemura et al., 1998). However, the detailed molecular mechanisms remain to be established.

Here we show that the forced repressive form of Xhairy2b is unable to induce the formation of secondary body axis and the expression of such organizer marker genes as follistatin, negating our prediction that the derepression mechanism would be responsible for the induction by Xhairy2b. Instead, the C-terminal WRPW
A
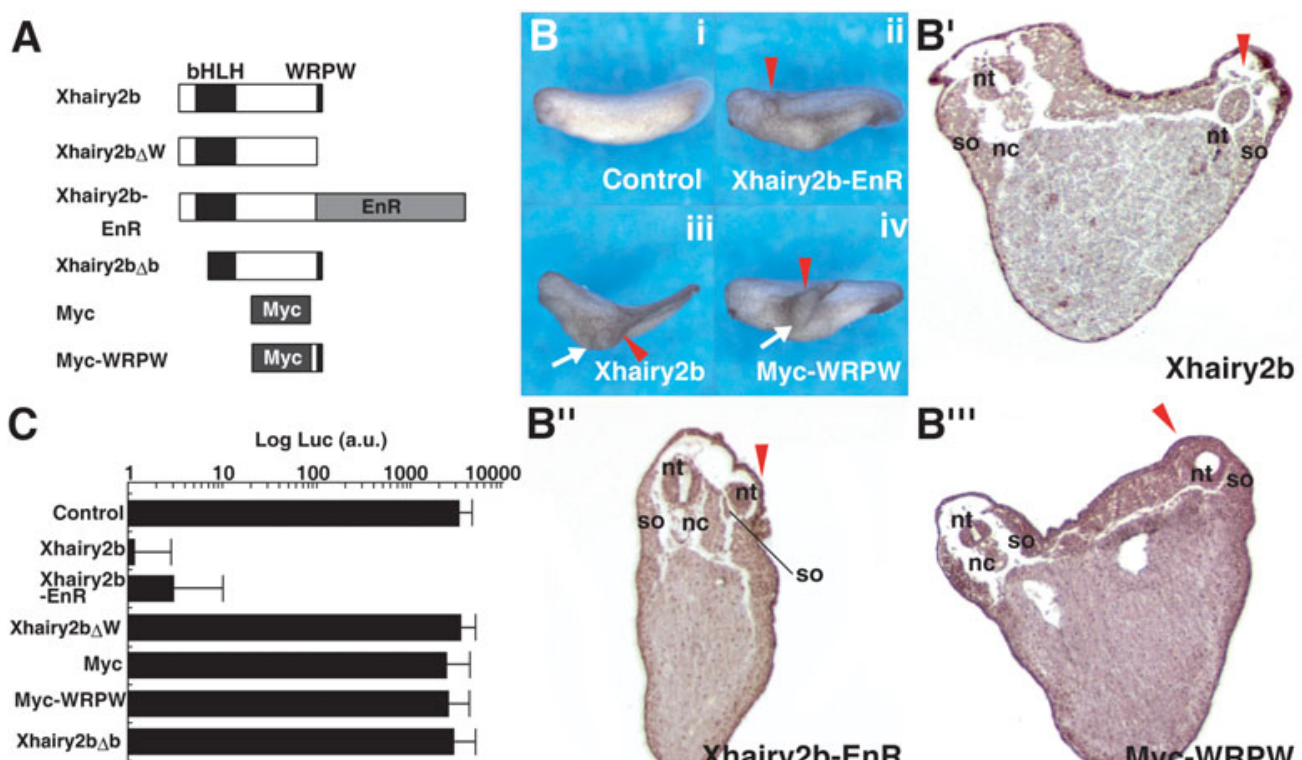
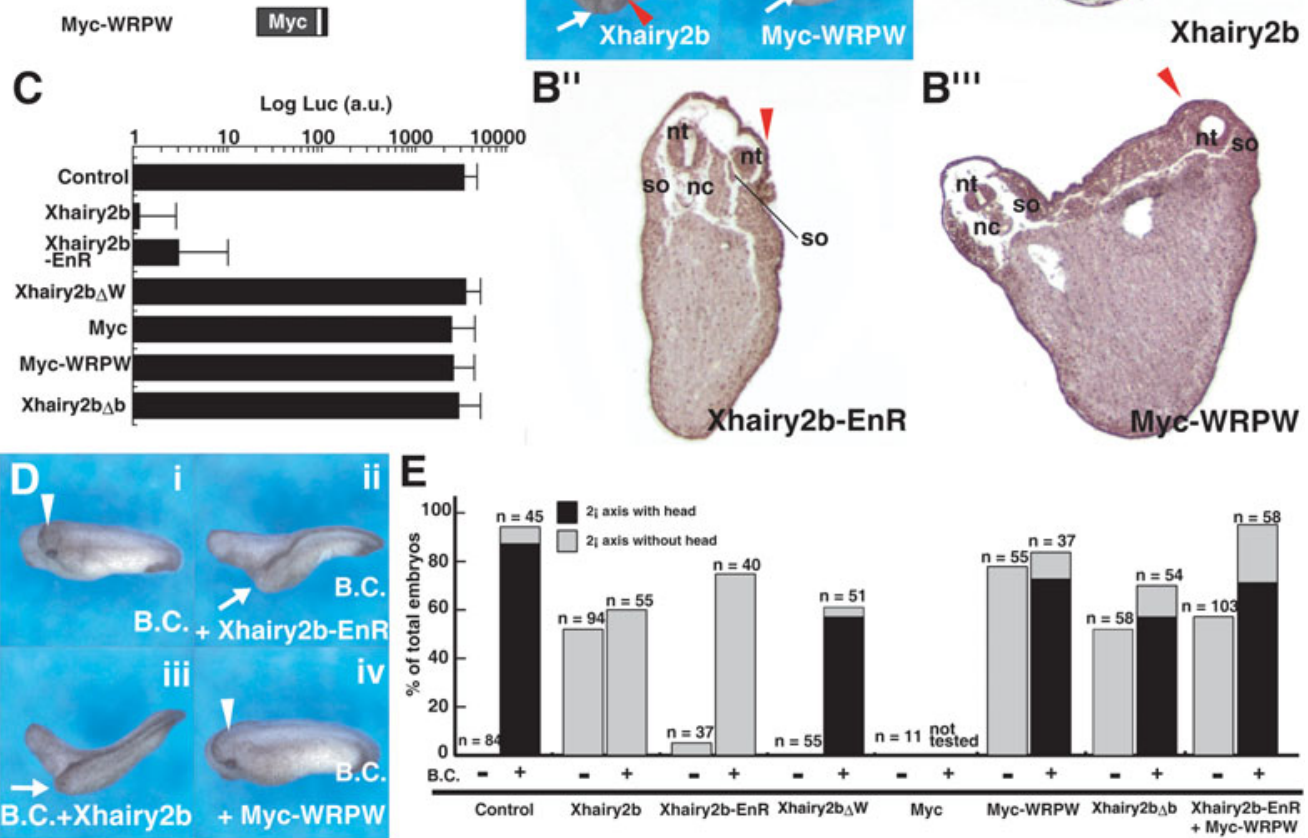

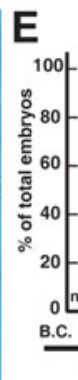

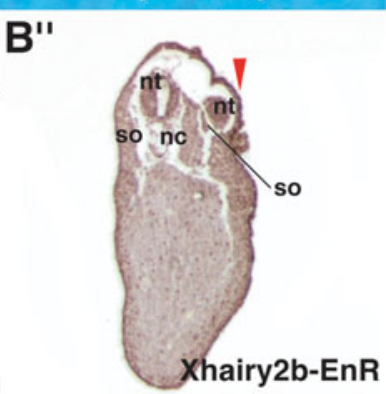

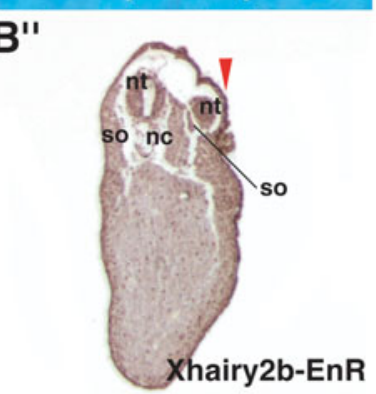

Xhairy2b-EnR

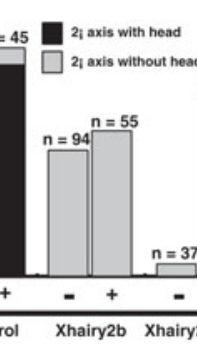

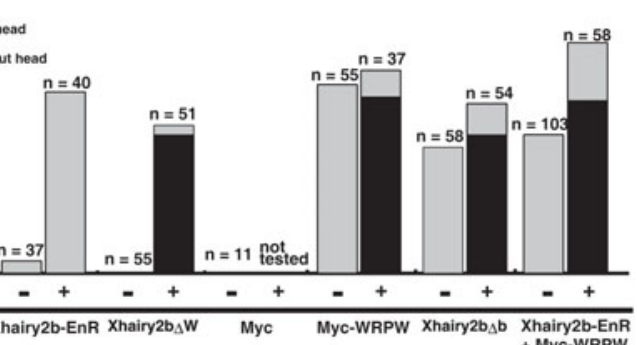

Xhairy2b-EnR
Fig. 1. Structures and functional properties of two Xhairy2b variants Xhairy2b-EnR and Myc-WRPW in comparison to the wild type. (A) Structures of Xhairy2b constructs. Top, wild type; Xhairy2buW, the C-terminal WRPW motif was deleted; Xhairy $2 b-$ EnR, the C-terminal 8 amino acid residues were replaced with Engrailed repression domain; Xhairy2bub, DNA-binding basic arm was deleted; Myc, six repeats of Myc epitope tags; MycWRPW, the C-terminal 8 amino acid residues containing the WRPW motif were $C$-terminally fused to 6 repeats of Myc epitope tag (indicated by Myc) so that all domains, such as the DNA binding basic arm and the dimerizing $\mathrm{HLH}$ domain, were deleted. bHLH, basic helix-loop-helix; EnR, Engrailed repression domain. (B i-iv) Axis induction by ventral expression of Xhairy $2 b$ and its variants was analyzed at stage 28 in comparison with uninjected control (i) 800 pg of Xhairy2b-EnR (ii), Xhairy2b (iii), or Myc-WRPW (iv) mRNA was injected into the ventral marginal zone of 4- to 8cell-stage embryos. The injection of Xhairy2b or Myc-WRPW mRNA induced the formation of secondary axes without head structures (arrows), whereas the injection of Xhairy2b-EnR mRNA resulted in small swelling. (B'-B'"') Transverse sections of the embryos shown in

(B). Red arrowheads indicate the corresponding secondary axes or swelling shown in (B ii-iv). Secondary axes induced by Xhairy2b (B') or Myc-WRPW (B'"') have neural tube and somitic mesoderm but lack notochord, while swelling caused by Xhairy2b-EnR (B") consists of neural tube and quite smaller mesodermal tissues, lacking notochord. nt, neural tube; nc, notochord; so, somite. (C) Transcriptional regulation via $\mathrm{N}$-box elements by variants shown in (A) was analyzed with $N$-box containing luciferase reporter vector driven by $\beta$-actin promoter. Embryos injected with the reporter vector alone served as control. Error bars indicate standard deviation $(n=10)$. (D) Head repression by ventral co-expression with $\beta$-catenin $m R N A$ was analyzed with either Xhairy2b, Xhairy2b-EnR, or Myc-WRPW mRNA. Injection was performed as described in (B) except that 80 pg of $\beta$-catenin mRNA was co-injected. The injection of $\beta$-catenin mRNA alone (i) or with Myc-WRPW mRNA (iv) formed complete secondary axes including head structures (arrowheads), whereas the co-injection with Xhairy2b (iii) or Xhairy2b-EnR (ii) mRNA caused head repression in the $\beta$-catenin induced secondary axes (arrows). B. C., $\beta$-catenin. (E) Summary of the frequencies of axis induction and head repression by each constructs shown in $(A)$ in the presence (B. $C$. + ) or absence of $\beta$-catenin (B.C.-). For each construct, 800 pg mRNA was injected as described in (B), while 80 pg of $\beta$-catenin mRNA was further added when testing the head-inhibition. The data of three representative experiments out of at least five independent experiments were combined. $2^{\circ}$ axis, secondary body axis; Xhairy2b-EnR + Myc-WRPW, co-expression of Xhairy2b-EnR and Myc-WRPW mRNA (800 pg each). 

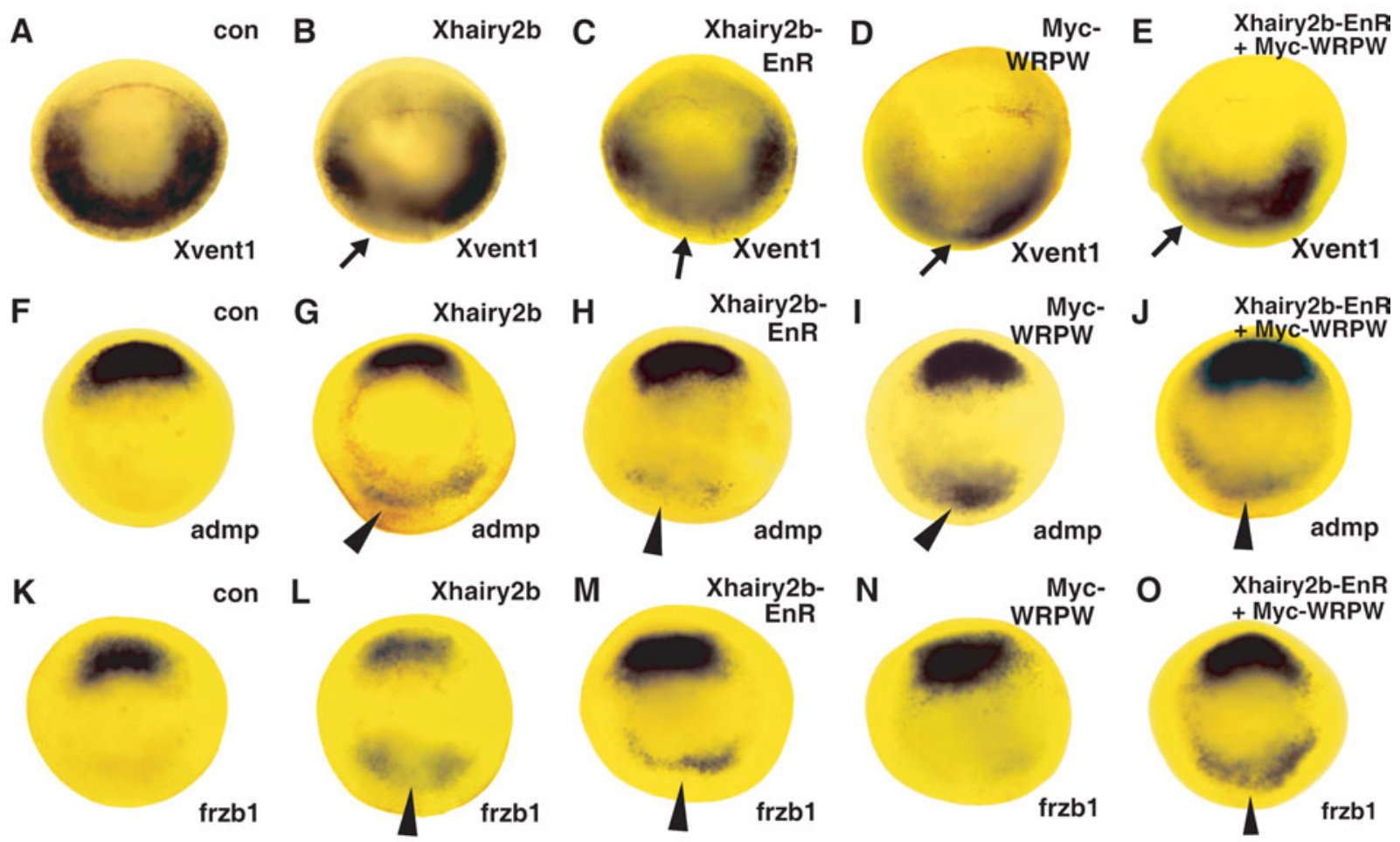

Fig. 2. Effects of Xhairy2b, Xhairy2b-EnR and Myc-WRPW overexpression on ventral and organizer marker genes at stage 10.5. (A-O) $m R N A$ of either Xhairy2b (800 pg), Xhairy2b-EnR (800 pg), Myc-WRPW (800 pg), orXhairy2b-EnR and Myc-WRPW (800 pg +800 pg) was injected into the ventral marginal zone of 4- to 8-cell-stage embryos and the embryos were fixed at stage 10.5 for whole-mount in situ hybridization; shown in vegetal view with dorsal side up; arrows indicate no or reduced expression and arrowheads indicate ectopic induction. The injected mRNA is indicated at the upper right corner of each panel. The ventral marker analyzed was (A-E) Xvent1. Organizer markers analyzed were (F-J) admp and (K-O) frzb1. (A), (F) and (K) show un-injected controls. Embryos were counterstained with Bouin's Fixative (yellow) and cleared with Murray's solution for ease of signal detection.

tetrapeptide motif solely exerted the inductive ability of Xhairy2b, which is clearly independent of transcriptional regulation. The coexpression of the repressive form and WRPW led to the successful reconstruction of the wild-type Xhairy2b function. Interestingly, WRPW alone induced the expression of genes specific for the anterior endoderm that Xhairy2b represses. Based on these results, we propose that Xhairy2b has two modes of action: the activity of WRPW intrinsically induces the expression of genes specific for the organizer in a rather non-specific manner to ensure the organizer environment. Then, the transcriptional repression selectively down-regulates the expression of some of these genes, resulting in the regionalization of the axial mesoderm. Our study provides new insight into how a region of the vertebrate embryo is demarcated by one dual-functional transcription factor in the early stages of development.

\section{Results}

The forced repressive form of Xhairy2b, Xhairy2b-EnR, mimics the repressive character of Xhairy2b but loses inductive ability

Although Xhairy2b is thought to be a transcriptional repressor judging from its primary structure (Tsuji et al., 2003), recent studies on bHLH transcription factors have suggested that the molecular mechanisms of the bHLH transcription factors are not always limited to direct transcriptional regulation (e.g., Bae et al., 2000; Sun et al., 2001; Giagtzoglou et al., 2003). Therefore, we first examined whether the known functions of Xhairy2b on the demarcation of the Spemann-Mangold organizer are indeed attributed to the transcriptional repression. To this end, we tested two variants that were unable to repress transcription via $\mathrm{N}$-box (Xhairy2b $\Delta \mathrm{b}$ and $\mathrm{Xhairy} 2 \mathrm{~b} \Delta \mathrm{W}$ ) and one forced repressive variant (Xhairy2b-EnR, see Fig. 1A, C and their legends for these three variants).

As one of its important functions, Xhairy2b inhibits head formation by down-regulating the expression of genes that are involved in head formation. To determine if the head-inhibition required transcriptional repression, we ventrally injected $X$ hairy2 $b$,

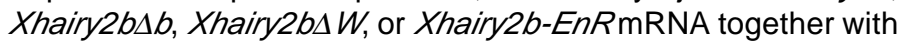
$\beta$-catenin mRNA (Yamaguti et al., 2005). The formation of the complete head structures induced by $\beta$-catenin mRNA injection (secondary body axis with head $87 \%$, without head $7 \%, n=45$, Fig. 1Di, E) was strongly repressed by the addition of Xhairy2bEnR mRNA (secondary body axis with head $0 \%$, without head $75 \%, n=40$, Fig. $1 \mathrm{Bii}, \mathrm{E})$ in a manner similar to that of wild-type Xhairy $2 b$ (secondary body axis with head $0 \%$, without head $60 \%$, $\mathrm{n}=55$, Fig. 1Biii, E). As expected, Xhairy2b $\Delta \mathrm{b}$ and Xhairy2b $\Delta \mathrm{W}$ were unable to inhibit head formation induced by $\beta$-catenin (Xhairy2b $\Delta \mathrm{b}$ : secondary body axis with head $56 \%$, without head $13 \%, n=54$; Xhairy $2 b \Delta \mathrm{W}$ : secondary body axis with head $57 \%$, without head $4 \%, n=51$; see Fig. $1 E$ ). Collectively, the results in head-inhibition were consistent with our prediction that the transcriptional repression would account for the functions of Xhairy2b.

We next examined the other function of Xhairy2b, namely axis 
TABLE 1

SUMMARY OF WISH FOR ECTOPIC INDUCTION OF MESODERMAL AND ANTERIOR MARKERS

\begin{tabular}{|c|c|c|c|c|}
\hline Injected mRNA & Xhairy2b & Xhairy2b-EnR & Myc-WRPW & $\begin{array}{l}\text { Xhairy2b-EnR } \\
+ \text { Myc-WRPW }\end{array}$ \\
\hline & $\begin{array}{c}\text { ectopic } \\
\text { induction (\%) }\end{array}$ & $\begin{array}{c}\text { ectopic } \\
\text { induction (\%) }\end{array}$ & $\begin{array}{c}\text { ectopic } \\
\text { induction (\%) }\end{array}$ & $\begin{array}{c}\text { ectopic } \\
\text { induction (\%) }\end{array}$ \\
\hline admp & $++(60 \%, n=60)$ & $+(23 \%, n=40)$ & $+++(80 \%, n=30)$ & $+++(90 \%, n=20)$ \\
\hline chd & $++(55 \%, n=55)$ & $-(0 \%, n=37)$ & $+++(83 \%, n=30)$ & $++(67 \%, n=30)$ \\
\hline follistatin & $++(43 \%, \mathrm{n}=89)$ & $-(0 \%, \mathrm{n}=26)$ & $+++(78 \%, n=41)$ & $+++(72 \%, \mathrm{n}=32)$ \\
\hline frzb1 & $+++(78 \%, n=78)$ & $+(36 \%, n=28)$ & $-(9 \%, n=47)$ & $+++(74 \%, n=19)$ \\
\hline Xdkk1 & $-(7 \%, n=62)$ & $+(32 \%, n=28)$ & $+++(71 \%, \mathrm{n}=35)$ & $+(30 \%, n=30)$ \\
\hline $\mathrm{X} \lim 1$ & $-(2 \%, n=56)$ & $-(9 \%, n=22)$ & $+++(70 \%, n=35)$ & $-(0 \%, n=20)$ \\
\hline Xotx2 & $-(0 \%, n=56)$ & $-(0 \%, n=19)$ & $++(50 \%, n=20)$ & $+(32 \%, n=19)$ \\
\hline Xhex & $-(0 \%, n=45)$ & $-(0 \%, \mathrm{n}=20)$ & $+++(77 \%, \mathrm{n}=35)$ & $-(0 \%, n=19)$ \\
\hline
\end{tabular}

Note. Signs representing the frequency of induction are defined as follows: - indicates $<15 \%$; + indicates $15 \leq 39 \%$; ++ indicates $40 \leq 69 \%$; and +++ indicates $\geq 70 \%$.

induction. Although Xhairy2b induced remarkable secondary body axes without head structures $(52 \%, n=94$, Fig. 1Biii, E; see also Fig. 1B' and its legend for histological analysis), the Xhairy2bEnR mRNA injected embryos formed swollen-like weak, if any, secondary body axes $(5 \%, n=37$, Fig. 1Bii, E; see also Fig. 1B" and its legend for histological analysis). Moreover, though ventral expression of Xhairy $2 \mathrm{~b} \Delta \mathrm{W}$ resulted in no axis-induction $(\mathrm{n}=55$; Fig. 1E) as expected, we found that Xhairy2bsb did not lose the axis-inducting activity when expressed ventrally (secondary body axis $52 \%, n=58$; Fig. $1 \mathrm{E}$ ).

The results shown above suggest that head-inhibition requires the repressive activity of Xhairy2b as a transcriptional repressor. However, the transcriptional repression would not be all about the known functions of Xhairy2b in that Xhairy2b-EnR lost the inductive functions. Also, though Xhairy $2 \mathrm{~b} \Delta \mathrm{W}$ totally lost the known functions of the wild-type, Xhairy $2 b \Delta b$ still kept the inductive activity (see below).

To investigate which function of Xhairy2b required transcriptional repression in molecular detail by comparing effects of Xhairy2b and Xhairy2b-EnR, we first checked if Xhairy2b-EnR shows the same behavior in the regulation of several marker gene

TABLE 2

\section{SUMMARY OF WISH FOR REPRESSION OF ANTERIOR, CHORDAMESODERMAL AND VENTRAL MARKERS}

\begin{tabular}{|c|c|c|c|c|}
\hline \multirow[t]{2}{*}{ Injected mRNA } & $\begin{array}{c}\beta \text {-catenin } \\
+ \text { Xhairy2b } \\
\end{array}$ & $\begin{aligned} & \beta \text {-catenin } \\
&+ \text { Xhairy2b-EnR } \\
&\end{aligned}$ & $\begin{aligned} & \beta \text {-catenin } \\
&+ \text { Myc-WRPW } \\
&\end{aligned}$ & $\begin{array}{c}\quad \beta \text {-catenin } \\
+ \text { Xhairy2b-EnR } \\
+ \text { Myc-WRPW }\end{array}$ \\
\hline & $\begin{array}{l}\text { no or reduced } \\
\text { expression (\%) }\end{array}$ & $\begin{array}{l}\text { no or reduced } \\
\text { expression (\%) }\end{array}$ & $\begin{array}{c}\text { no or reduced } \\
\text { expression }(\%)\end{array}$ & $\begin{array}{l}\text { no or reduced } \\
\text { expression (\%) }\end{array}$ \\
\hline chd & $+++(70 \%, n=57)$ & $++(51 \%, n=29)$ & $-(11 \%, n=19)$ & $++(42 \%, n=24)$ \\
\hline Xdkk1 & $+++(74 \%, n=78)$ & $+++(75 \%, n=28)$ & $-(10 \%, n=10)$ & $++(60 \%, n=30)$ \\
\hline Xlim1 & $+++(100 \%, n=49)$ & $+++(100 \%, n=40)$ & $-(10 \%, n=10)$ & $+++(95 \%, n=20)$ \\
\hline Xotx2 & $+++(100 \%, n=40)$ & $+++(97 \%, n=30)$ & $-(13 \%, n=8)$ & $+++(70 \%, \mathrm{n}=20)$ \\
\hline Xhex & $+++(92 \%, n=78)$ & $++(54 \%, n=28)$ & $-(0 \%, n=9)$ & $++(67 \%, n=30)$ \\
\hline \multirow[t]{2}{*}{ Injected mRNA } & Xhairy2b & Xhairy2b-EnR & Myc-WRPW & $\begin{array}{l}\text { Xhairy2b-EnR } \\
\text { +Myc-WRPW }\end{array}$ \\
\hline & $\begin{array}{c}\text { no or reduced } \\
\text { expression (\%) }\end{array}$ & $\begin{array}{l}\text { no or reduced } \\
\text { expression (\%) }\end{array}$ & $\begin{array}{l}\text { no or reduced } \\
\text { expression }(\%)\end{array}$ & $\begin{array}{l}\text { no or reduced } \\
\text { expression (\%) }\end{array}$ \\
\hline Xvent1 & $+++(77 \%, n=44)$ & $+++(80 \%, n=30)$ & $+++(87 \%, n=15)$ & $+++(70 \%, \mathrm{n}=20)$ \\
\hline
\end{tabular}

Note. (1) The frequency of induction by $\beta$-catenin alone is as follows: chd $(91 \%, n=32), X d k k 1(91 \%, n=35)$,

$X \lim 1(92 \%, n=38)$, Xotx2 $(96 \%, n=24)$, and Xhex $(93 \%, n=42)$. (2) Signs representing the frequency of repression

are defined as follows: - indicates $<15 \%$; + indicates $15 \leq 39 \%$; ++ indicates $40 \leq 69 \%$; and +++ indicates $>70 \%$. expressions as wild-type Xhairy2b does. First, to test whether the expression of ventral marker gene Xvent1 (Gawantka et al., 1995), which is down-regulated by Xhairy2b (Yamaguti et al., 2005), was affected, we ventrally injected Xhairy2b or Xhairy2b-EnR mRNA, followed by wholemount in situ hybridization (WISH). As expected, both Xhairy2b and Xhairy2b-EnR down-regulated the expression of Xvent1 (77\% repression, $n=44$, Fig. $2 B ; 80 \%$ repression, $n=30$, Fig. $2 C$, respectively).

Next, we investigated effects of Xhairy2b or Xhairy2b-EnRon the expression of anterior marker genes. As expected from the observations on head-inhibition (Fig. 1D, $\mathrm{E})$, the results of WISH clearly showed that the ectopic expression of $X d k k 1(91 \%, n=$ 35, Fig. 3B; Glinka et al., 1998), Xhex (93\%, $n=42$, Fig. 3H; Newman et al., 1997), Xotx2(96\%, $n=24$, Fig. 3N; Blitz and Cho, 1995) and $X \lim 1$ (92\%, $n=38$, Fig. 3T; Taira et al., 1992) induced by $\beta$-catenin was repressed by the co-expression of Xhairy2bEnR (Xdkk1, 75\% repression, $\mathrm{n}=28$, Fig. 3D; Xhex, $54 \%$ repression, $n=28$, Fig. 3J; Xot 2 , 97\% repression, $n=30$, Fig. 3P; (100\% repression, $\mathrm{n}=40$, Fig. $3 \mathrm{~V})$, in a manner similar to Xhail2b (Xdkk1, 74\% repression, $\mathrm{n}=78$, Fig. 3C; Xhex, 92\% repression, $n=78$, Fig. 3I; Xotx2, 100\% repression, $n=40$, Fig. O; Xlim1, 100\% repression, $n=49$, Fig. 3U)

Finally, we checked if Xhairy2b-EnR induces the expression of orker genes that Xhairy2b induces, such as follistatin (43\%, $n=89$, not shown; Hemmati-Brivanlou et al., 1994), admp (60\%, $n=60$, Fig. 2G; Moos et al., 1995), frzb1 (78\%, $n=78$, Fig. , Leyns et al., 1997; Wang et al., 1997) and chd $(55 \%, n=60$, phenotype (Fig. 1B, E), Xhairy2b-EnR never up-regulated the expression of follistatin $(n=26$, not shown) or $c h d(n=37$, Fig. 4D), admp (23\%, n= 40, Fig. $2 \mathrm{H})$ and frzb1 $(36 \%, \mathrm{n}=28$, Fig. $2 \mathrm{M}$ ) expression was induced weakly. The results of WISH analyses were summarized in Table 1 and Table 2.

Taken together, we concluded that Xhairy2b-EnR as a transcriptional repressor carries only one intrinsic function of wild-type Xhairy2b: the repression of genes that are expressed in neighboring tissues.

\section{The C-terminal WRPW motif alone is responsible for the inductive function of Xhairy2b}

The findings that Xhairy2b-EnR, but not Xhairy $2 b \Delta b$, is unable to induce the axial structure and to up-regulate corresponding marker gene expression indicate that other molecular mechanisms, independent of transcriptional repression characteristic of a HES protein, are required for the inductive activity of Xhairy2b. The critical difference in primary structure between axisinducible constructs (i.e., Xhairy2b and Xhairy $2 b \Delta b$ ) and axis-not-inducible con- 
A

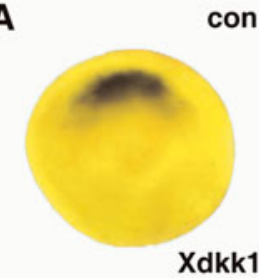

G

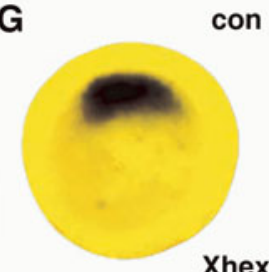

B

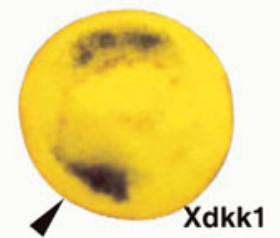

B.C. $\mathrm{C}$

(B) + Xhairy2b

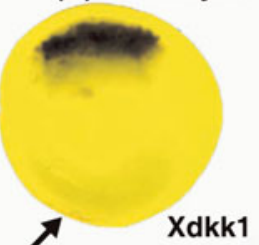

B.C. I
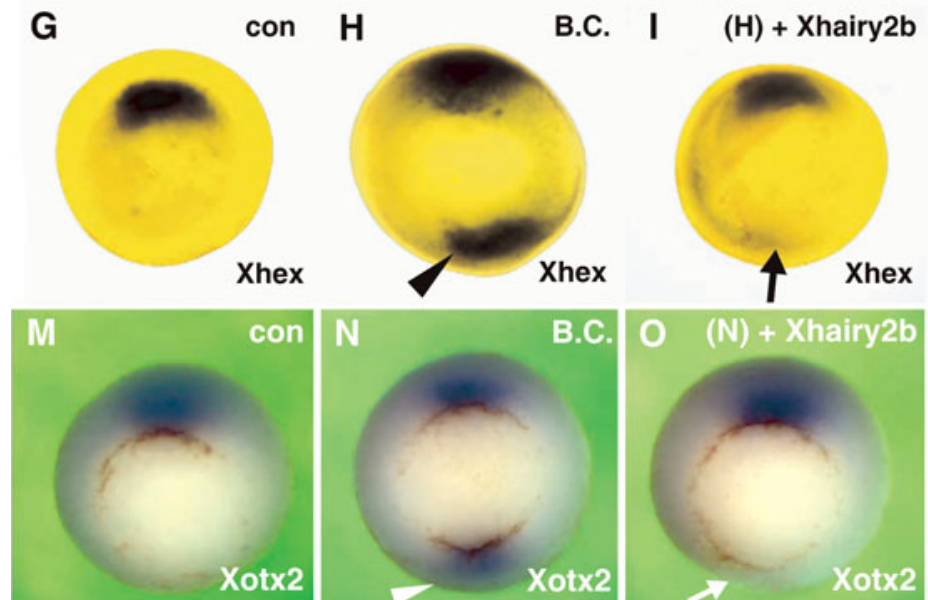

B.C.

otx2
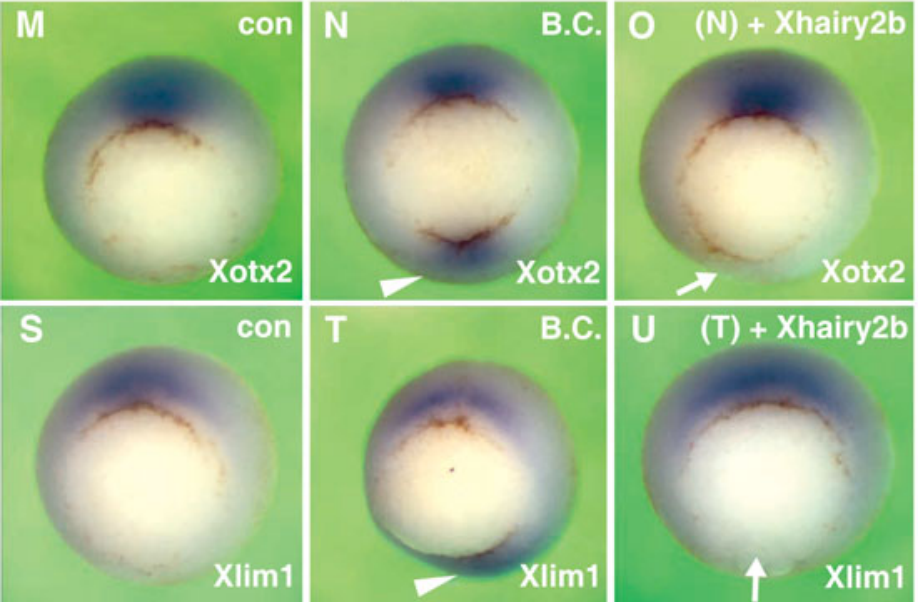

D
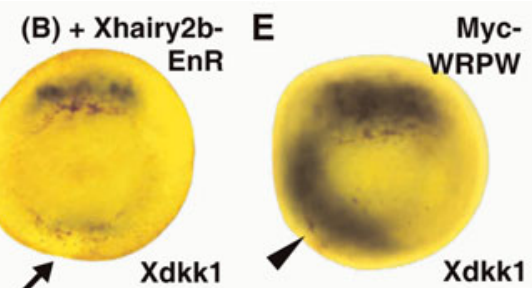

Myc- $F \quad(B)+$ Xhairy2b-EnR

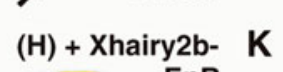

\section{$\mathbf{J}$}
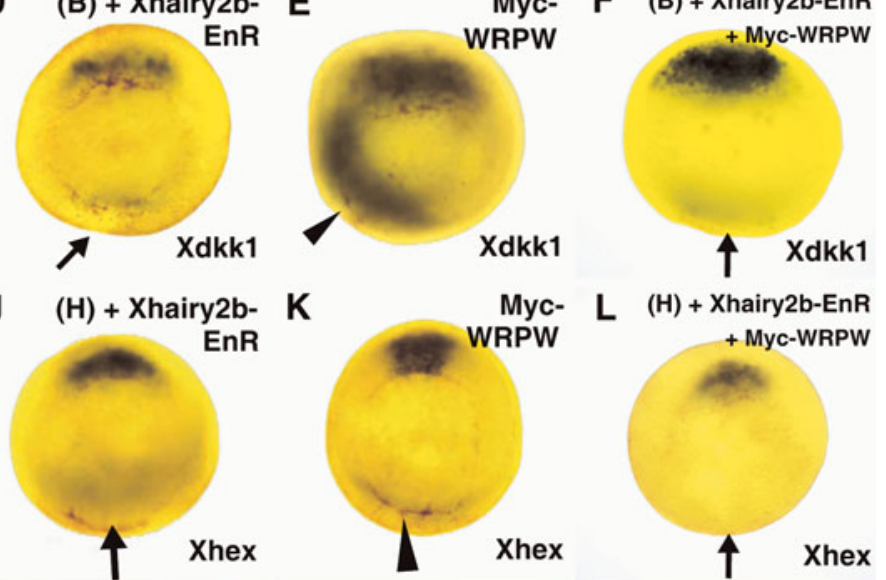

L $(\mathrm{H})+$ Xhairy2b-EnR
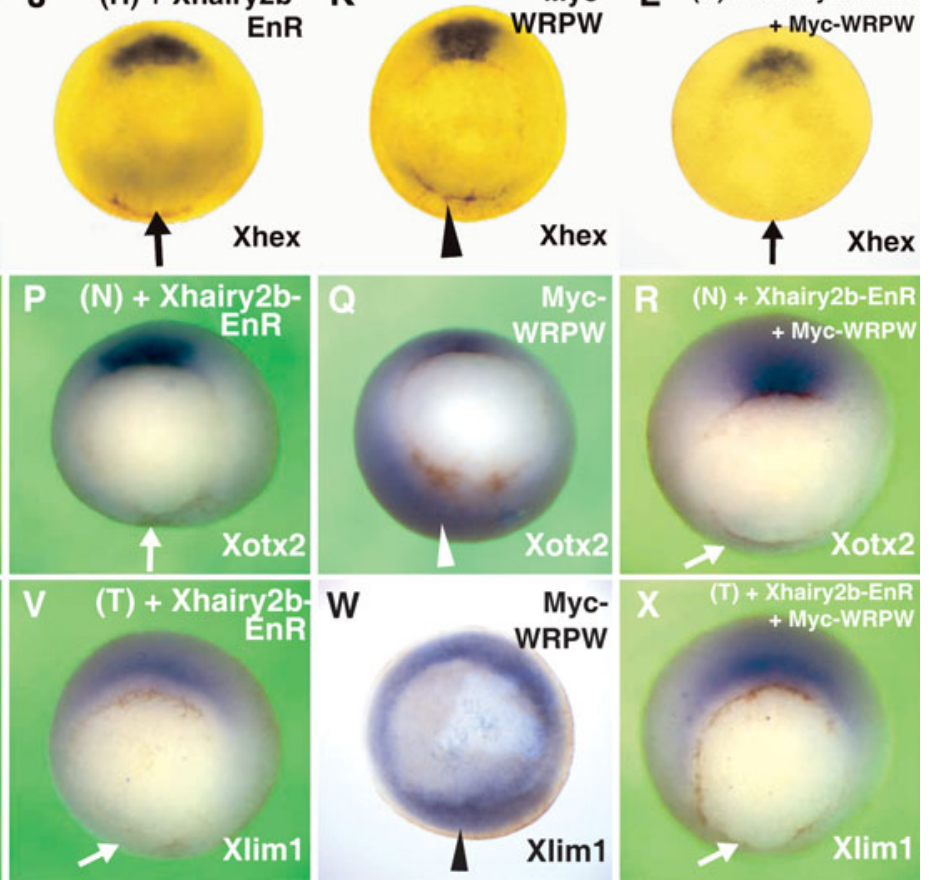

Fig. 3. Effects of Xhairy2b, Xhairy2b-EnR and Myc-WRPW overexpression on anterior endoderm marker genes at stage 10.5. (A-X) $m R N A$ for eitherXhairy2b (800 pg), Xhairy2b-EnR (800 pg), or Xhairy2b-EnR and Myc-WRPW (800 pg + 800 pg) together with $\beta$-catenin mRNA (80 pg) or mRNA for Myc-WRPW (800 pg) alone was injected into the ventral marginal zone of 4- to 8-cell-stage embryos and the embryos were fixed at stage 10.5 for whole-mount in situ hybridization; shown in vegetal view with dorsal side up; arrows indicate no or reduced expression and arrowheads indicate ectopic induction. The injected mRNA is indicated at the upper right corner of each panel. Marker genes analyzed were (A-F) Xdkk1, (G-L) Xhex, (MR) Xotx2 and (S-X) Xlim1. (A), (G), (M) and (S) show uninjected controls. Embryos shown in (A-L) were counterstained with Bouin's Fixative (yellow) and (A-L, W) were cleared with Murray's solution. Note that Myc-WRPW alone induced the ectopic expression of these anterior marker genes. For the data of co-injection of Myc-WRPW and $\beta$-catenin or of the same markers when $\beta$-catenin mRNA was not co-injected, see Tables 1 and 2.

structs (i.e., Xhairy2b-EnR and Xhairy2b $\Delta \mathrm{W}$ ) is the presence or absence of the WRPW motif at their carboxyl terminus. This fact prompted us to assume that the WRPW motif might solely have the inductive ability of Xhairy2b.

For direct examination of the role of WRPW motif in the inductive function of Xhairy2b, we constructed Myc-WRPW by fusing 6 repeats of Myc epitope tag with the WRPW motif (Fig. 1A). As expected, Myc-WRPW as well as Myc tags alone were unable to repress the transcription via the N-box (Fig. 1C). However, interestingly, we found that the ventral expression of Myc-WRPW resulted in induction of the secondary body axis without head structures (78\%; secondary body axis with head $0 \%$, $\mathrm{n}=55$, Fig. 1Biv, E; see also Fig. B"' for histological analyses comparison with $\mathrm{B}^{\prime}$ and $\mathrm{B}^{\prime \prime}$ ), but head formation was never inhibited by the Myc-WRPW when co-expressed with $\beta$-catenin (secondary body axis with head $73 \%$, without head $11 \%, \mathrm{n}=37$, Fig. 1Div, E) as expected. Since Myc tags alone did not induce the secondary body axes (normal development $100 \%, n=11$, Fig.
$1 \mathrm{E})$, it was strongly suggested that WRPW motif might carry the inductive functions of Xhairy2b. This conclusion is further supported by the fact that GFP-tagged WRPW behaved in a similar way to Myc-WRPW (data not shown).

We further investigate effects of Myc-WRPW on the marker genes tested with Xhairy2b and Xhairy2b-EnR by means of WISH. Consistent with the external phenotype, Myc-WRPW caused reduced expression of $X$ vent 1 (87\% repression, $n=15$, Fig. 2D). In addition, Myc-WRPW induced the ectopic expression of follistatin (78\%, $\mathrm{n}=41$, not shown), admp (80\%, $\mathrm{n}=30$, Fig. 2I) and $c h d(83 \%, n=30$, Fig. $4 \mathrm{E})$, although the induction of frzb1 was quite weak ( $9 \%, n=47$; Fig. $2 \mathrm{~N})$, summarized in Table 1. Also, when co-expressed with $\beta$-catenin, Myc-WRPW did not affect ectopic expression of the anterior marker genes (see Table 2). Interestingly, WRPW alone induced the expression of anterior markers that Xhairy2b repressed when expressed ventrally (Xdkk1, $71 \%$ induction, $\mathrm{n}=35$, Fig. 3E; $X$ hex, $77 \%$ induction, $\mathrm{n}=35$, Fig. $3 \mathrm{~K}$; Xotx2, 50\% induction, $\mathrm{n}=20$, Fig. 3Q; Xlim1, 70\% induction, 

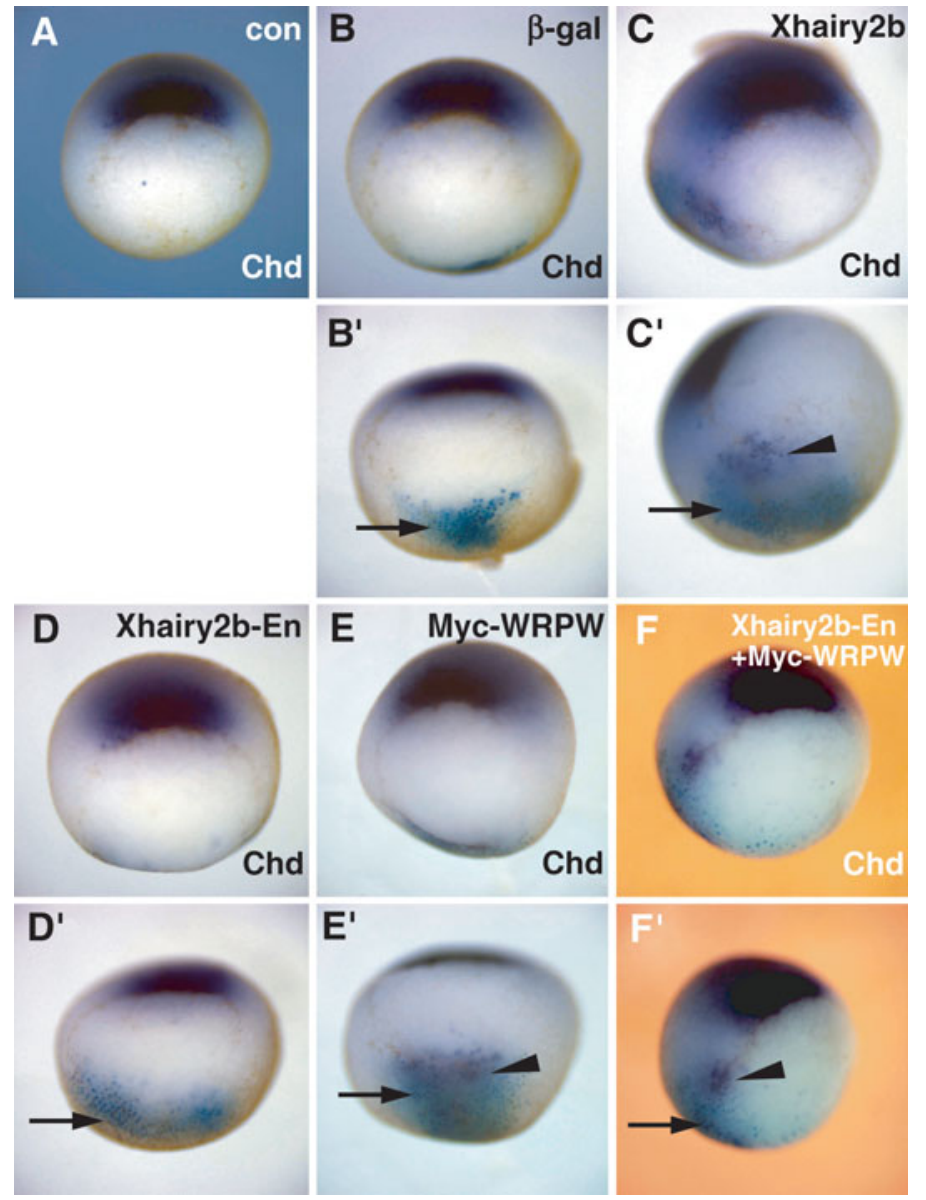

Fig. 4. Ectopic induction of chd expression by Xhairy2b and its variants. (A-F) mRNA for either Xhairy2b (800 pg), Xhairy2b-EnR $(800$ $\mathrm{pg})$, or Xhairy2b-EnR and Myc-WRPW $(800 \mathrm{pg}+800 \mathrm{pg})$ together with n$\beta$-gal mRNA (200 pg) or mRNA of n- $\beta$-gal mRNA (200 pg) alone was injected as described above, followed by whole-mount in situ hybridization at stage 10.5; shown in vegetal view with dorsal side up. The injected mRNA is indicated at the upper right corner of each panel. ('A'-F') show the same embryos in (A-F) respectively, but from a different orientation. Arrowheads indicate ectopic chd expression, whereas arrows indicate $m R N A$ injected region stained by $X$-gal. Note that the ectopic induction of chd expression (purple) by Xhairy2b ( $C^{\prime}$ ) or the co-expression of Xhairy2bEnR and Myc-WRPW $\left(\mathrm{F}^{\prime}\right)$ does not overlap with the injected site indicated by $X$-gal staining (turquoise).

$\mathrm{n}=35$, Fig. 3W; see Table 1 for summary). We thus concluded that the WRPW motif alone was in charge of the inductive part of Xhairy2b functions.

\section{Co-expression of Xhairy2b-EnR and Myc-WRPW reconstructs wild-type Xhairy2b function}

The functional differences between Xhairy2b-EnR and MycWRPW collectively imply that Xhairy2b might potentially upregulate the expression of genes in the Spemann-Mangold organizer through the activity of the WRPW motif, but its activity as a transcriptional repressor selectively down-regulates most of the gene expressions except those that ensure the dorsal environment, such as follistatinand chdexpression. If this were the case, the co-expression of Xhairy2b-EnR and Myc-WRPWwould functionally complement each other to reconstruct the functions of Xhairy2b.

To test this hypothesis, we ventrally co-injected Xhairy2b-EnR and $M y c-W R P W \mathrm{mRNA}$ (and $\beta$-catenin mRNA when testing the repression). The axis induction and head repression were recapitulated in the external phenotype of the co-injected embryos (secondary body axis with head $0 \%$, without head $57 \%, n=103$; Fig. 1E). Head suppression was slightly attenuated, as seen when $\beta$-catenin mRNA was further added (secondary body axis with head $71 \%$, without head $24 \%, n=58$; Fig. $1 \mathrm{E}$ ). We further conducted WISH analyses on the sets of genes examined so far in comparison with the wild type and two variants. As summarized in Tables 1 and 2, the results of WISH were in good agreement with those of Xhairy2b. For example, the expression of admp, follistatin, frzb1 and chdwas up-regulated by the co-expression of Xhairy2b-EnR and Myc-WRPW (admp 90\%, $\mathrm{n}=20$, Fig. 2J; follistatin $72 \%, \mathrm{n}=32$, not shown; frzb174\%, $\mathrm{n}=19$, Fig. 2O; chd $67 \%, n=30$, Fig. 4F), which Xhairy2b-EnR alone never or weakly up-regulated. Also, the repressive function of Xhairy $2 \mathrm{~b}$ on ventral and anterior marker gene expression was successfully recapitulated by the co-expression of Xhairy2b-EnR and Myc-WRPW (Xvent $1,70 \%$ repression, $\mathrm{n}=20$, Fig. $2 \mathrm{E} ; X d k k 1,60 \%$ repression, $\mathrm{n}=30$, Fig. 3F; Xlim1, 95\% repression, $\mathrm{n}=20$, Fig. 3X; Xot $2,70 \%$ repression, $\mathrm{n}=20$, Fig. $3 \mathrm{R}$; Xhex, $67 \%$ repression, $\mathrm{n}=30$, Fig. $3 \mathrm{~L}$ ). All in all, the results of co-expression strongly suggest that Xhairy $2 b$ functions via a combination of the two molecular mechanisms so that the broad inductive functions through the WRPW motif would be tapered by the selective down-regulation as a bHLH repressor in order to create a pattern of gene expression in the Spemann-Mangold organizer.

\section{Discussion}

In the present study, we investigated the molecular mechanism of Xhairy2b function by utilizing the fusion and deletion variants of Xhairy $2 b$ and showed that the inductive function of Xhairy $2 b$ is independent of transcriptional repression and carried out only through the C-terminal WRPW motif. Only transcriptional regulation is responsible for the repressive function. These results indicate that $\mathrm{Xhairy} 2 \mathrm{~b}$ is a dual-functional protein possessing another function independent of direct transcriptional regulation.

\section{The possible role of the WRPW motif in the inductive function of Xhairy2b}

We successfully characterized the inductive function of Xhairy2b as the activity through the WRPW motif alone that obviously lacks the ability to bind to DNA. In a similar manner, ascidian Pem1, which has no known DNA binding domain, possesses the WRPW motif at the C-terminus and is involved in anterior and dorsal pattern formation (Yoshida et al., 1996). Therefore, this particular motif could possibly act as an indirect transcriptional regulator. In addition, it is known that the C-terminal structure of mouse Hes 1 protein can modulate the transcriptional activity of the Runt related transcription factor Cbfa1 (McLarren et al., 2000). In this case, the DNA binding activity of the Hes1 protein is also not required (McLarren et al., 2000). Taken together, it is suspected that the inductive property of Xhairy2b might depend upon the 
protein-protein interaction of the WRPW portion with a component of a different transcriptional machinery.

\section{Synergistic effect of inductive and repressive activities of Xhairy2b}

We also found in Xhairy2b an intriguing relationship between its repressive and inductive activities, which was seen in frzb1 expression. frzb1 expression was weakly induced by either Xhairy2b-EnR (Fig. 2R) or Myc-WRPW (Fig. 2S), although Xhairy2b efficiently up-regulates the expression of frzb1 when expressed ventrally (Fig. 2Q). Similar to the wild-type Xhairy2b, the co-expression of Xhairy2b-EnR and Myc-WRPW efficiently induced the expression of frzb1 (Fig. 2T). Therefore, it is possible to assume that the synergistic effect of transcriptional repression and inductive activity of the WRPW motif would be required for the induction of frzb1. Together with the previous observation that frzb1, an antagonist of Wnt signaling, is predominantly expressed in the prechordal mesoderm (Leyns et al., 1997; Wang et al., 1997), overlapping with Xhairy2b expression (Tsuji et al., 2003; Yamaguti et al., 2005), our finding could inspire more detailed analyses of the establishment of the prechordal plate identity.

\section{Pattern formation through induction-repression-coupled mechanism by Xhairy2b}

The sequential combination of inductive and repressive gene regulation is a commonly adopted strategy for pattern formation in early vertebrate development. For example, in Xenopus, general mesoderm inducing signals induce the expression of the transcription factor Xbra (Smith et al., 1991), which has the ability to up-regulate goosecoidand Xvent2(Messenger et al., 2005), in the entire marginal zone (Harland and Gerhart, 1997). Since the ventral/ lateral specific BMP signals restrict the expression of Xvent2(Onichtchouk et al., 1996), a direct repressor of gsc, gsc expression becomes localized to the dorsal mesoderm. Gsc, in turn, directly represses the expression of Xbra (Artinger et al., 1997) and Xvent2 (Messenger et al., 2005). This sequential induction-repression-coupled mechanism plays an important role in regionalization and pattern formation.

Our findings suggest that Xhairy2b alone exerts these induction-repression regulations via the two modes of action. Then, what is the biological significance of the inductive and repressive functions being present in a single molecule? We found that Xhairy2b can induce follistatin expression in the same region in which Xhairy2b is ectopically expressed. chd expression was down-regulated in Xhairy2b-positive cells, but was ectopically induced in cells that surrounded the Xhairy2b-positive cells (Fig. 4). Furthermore, the expression of such ventral markers as Xvent1 was totally repressed in the ectopic Xhairy2b-positive cells, so that precise patterning to generate these three distinct regions is established based on Xhairy $2 b$ expression. Although several explanations can be given, our results lead us to propose the possible underlying mechanism: the WRPW portion of Xhairy2b functions in the induction of follistatinand chdexpression, whereas the repressor activity of Xhairy2b down-regulates the expression of chd and Xvent1.

How can this proposal explain the relationship between $X$ hairy2 $b$ and chdin normal development? Here we focus on the expression of Xhairy2band chdin late gastrula embryos. chdis expressed in the chordamesoderm, whereas Xhairy2b is expressed in the anterior prechordal mesoderm and in the overlying floor plate (Yamaguti et al., 2005); thus, chd expression is surrounded by Xhairy2b expression. A previous study has shown that initial selection is established in early gastrulae where Xhairy2a-expressing cells acquire a non-involuting nature, differentiating into floor plate cells, whereas chdexpressing cells involute during gastrulation to become notochordal cells (López et al., 2005). However, the mechanism to maintain these identities in late gastrulae was not clarified. Based on our observation that the ectopic chdexpression was induced not in Xhairy2b-positive cells but in the surrounding Xhairy2b-negative cells, it is possible to assume that some secretion factor could play a role in the induction or maintenance of chdexpression by WRPW-mediated Xhairy2b inductive function. Since the repressor activity of Xhairy2b down-regulates the expression of $c h d$, it is consistent that ectopic chdexpression was induced in Myc-WRPW-positive cells (Fig. 4) in which there would exist chd-inducing signals but repressors against chdexpression would not exist.

These results could imply that chd expression can be upregulated equally in the prechordal mesoderm, the chordamesoderm and the floor plate. In actuality, however, Xhairy $2 b$ represses the expression of $c h d$ in the prechordal mesoderm and the floor plate, resulting in the restriction of chdexpression to the chordamesoderm. In conclusion, a transcription factor with such two modes of action might be advantageous for "sharpening" positional information in that it requires fewer signals to establish the specific identity of a cell, although more detailed molecular mechanisms remain to be elucidated in future studies.

\section{Materials and Methods}

\section{Embryonic manipulation}

Xenopus laevis embryos were in vitrofertilized, dejellied and cultured as described (Hawley et al., 1995) and staged according to Nieuwkoop and Faber (1967). Embryos were fixed in MEMFA (Harland, 1991) at stage 10.5 for $\mathrm{WISH}$, or at stages $28-30$ for phenotype analyses. For histological analyses, embryos were fixed with MEMFA at the indicated stage, dehydrated with methanol, embedded in paraffin and sectioned in $10-\mu \mathrm{m}$ slices, followed by hematoxylin staining.

\section{Plasmid construction}

For the construction of $p B S$-Xhairy2b, cDNA of Xhairy2bwas synthesized with oligo-dT-Not1 primer (New England Biolabs). After second strand synthesis, a blunt-ended adaptor duplex (New England Biolabs) with 5'-EcoR1 linker was ligated with the double-stranded Xhairy2b cDNA. Then, the Xhairy2bcDNA was inserted into the EcoRI-Not1 sites in pBluescript KS+. To generate the $p B S$-Xhairy2b-EnR construct, a cDNA coding for the Engrailed2 repression domain (Poole et al., 1985) was PCR-amplified and inserted into $p B S$-Xhairy $2 b$ that was cut with Eco47III/Ncol sites. For $p X \beta m$-Xhairy2b $\Delta b, p B S$-Xhairy2 $b$ was digested with $\mathrm{EcoRI} / \mathrm{Notl}$, inserted in the EcoRI-Notl sites in $p X \beta m$ to generate $p X \beta m$-Xhairy $2 b$. Then, sequence upstream and downstream of the basic arm coding sequences of $p X \beta m$-Xhairy $2 b$ was PCR amplified with the following primer sets, respectively:

\section{F1 5'ATGCCTGCAGATAGTATGGAGAA \\ R1 5'GGCACTCTTGGGTTTATCCG \\ F2 5'GAGCGCGAATCAACGAGAGC \\ R2 5'CTGCAGGTTCCGTAGG.}

After digesting the fragments with Hinfl, the two fragments were ligated. The sequence of the ligated fragements were further PCRamplified with F1-R2 primer sets, followed by Pstl digestion. The digested fragment was inserted into the Pstl-disgested $p X \beta m$-Xhairy2b. To gener- 
ate $p B S$-Xhairy2b $\Delta W, p B S$-Xhairy2bwas cut with Eco47III/Ncol sites and blunted, followed by self-ligation. $p C S 2 A T+-M y c-W R P W$ was constructed as described previously (Tsuji and Hashimoto, 2005). pCS2+- $\beta$-catenin was a kind gift from Dr. David Turner.

Plasmids containing marker genes used in this study were as follows. Using forward and reverse primers based on the published sequence, the coding region of each gene was amplified by RT-PCR. The sequences of the primers are shown below.

admp:

F 5'GCCCATCGATCCACCATGGACCTTAGGAAGATGTTGGG R 5'GCCCCTCGAGTTAGTGGCACCCGCAGCTGC frzb1:

F 5'CCCGAATTCCACCATGTCTCCAACAAGGAAATTGGAC R 5'CCCGGCGCGCCCTAACTACGCGCTTGTCTGGAATT Xdkk1:

F 5'CCCGAATTCCACCATGTCTCCAACAAGGAAATTGGAC R 5'CCCGGCGCGCCCTAACTACGCGCTTGTCTGGAATT $X \lim 1:$

F 5'CCCGAATTCCACCATGGTTCACTGTGCTGGATGCG R 5'CCCGGCGCGCCCTACCACACTGCCGTTTCGTTC Xvent1:

F 5'CCCGAATTCCACCATGGTTCAACAGGGATTCTCTATTG R 5'CCCGGCGCGCCTTACATATACTGAGCCCCAAAGAG and Xhex:

F 5'CCCGAATTCCACCATGCAGTACCAGCACCCCAGCTCCTC R 5'CCCGGCGCGCCTTAATGTGCACAGTTGTAATATCCTTTGTCG

These PCR products were digested with EcoR1/Asc1 (frzb1, Xdkk1, Xlim1, Xvent1 and Xhex) or Cla1/Xho1 (admp) and ligated into the pCS2AT + that was constructed by insertion of annealed oligonucleotides (5'TCGAGGGCGCGCCGATATCTCTAGACGCCCTATAGTGAGTCGTATTAC3' and 5'GTAATACGACTCACTATAGGGCGTCTAGAGATATCGGCGCG CCC3') into Xhol-SnaBI digested pCS2+. This creates new Ascl and EcoRV sites in the polylinker I region.

\section{Microinjection}

Capped mRNAs for microinjection were synthesized from the linearized plasmids by using the mMESSAGE mMACHINE kit (Ambion). For $X$ hairy $2 b$ and $X$ hairy2b $\Delta W$, the plasmid was linearized with Notl and transcribed with T3 polymerase. For Xhairy2b-EnR, the plasmid was linearized with Sacll and transcribed with T3 polymerase. For Xhairy2b $\Delta b$, Myc (pCS2-MT), Myc-WRPW and $\beta$-catenin, the plasmids were linearized with Notl and transcribed with SP6 polymerase.

mRNA and/or expression plasmid was microinjected into the ventral marginal zone of 4- to 8-cell-stage embryos at the indicated doses. To test the effects of the co-expression of Xhairy2b-EnR and Myc-WRPW, the effects of each variant alone and wild-type Xhairy2b were also tested by using siblings from the same parents. Except for reporter assays, $800 \mathrm{pg}$ of EYFP(Clontech) mRNA was co-injected to confirm the injected region.

\section{Whole-mount in situ hybridization (WISH)}

WISH was performed as described previously (Harland, 1991) with minor modifications. To remove pigments of embryos, $6 \% \mathrm{H}_{2} \mathrm{O}_{2}$ in PBST buffer was used. To detect admp, frzb1, Xvent1, Xdkk1 and Xhex expression, samples were postfixed with Bouin's Fixative (yellow) without subsequent washes and cleared with Murray's solution (Mizuseki et al., 1998).

\section{Luciferase reporter assay}

Luciferase reporter assay was performed with the Dual-Luciferase Reporter Assay System (Promega) and a luminometer (TD-20/20, TURNER DESIGNS). Reporter plasmid PGV-B-N6-LUC was a kind gift from Dr. Ryoichiro Kageyama. Briefly, 6 repeats of $\mathrm{N}$-box element (CACGAG) were followed by $\beta$-actin promoter and firefly /uciferase coding sequence. Embryos were ventrally un-injected or injected with 800 $\mathrm{pg}$ of mRNA to be tested together with $240 \mathrm{pg}$ of reporter plasmid at the
4- to 8-cell stage. The embryos were cultured and harvested for measurement of luciferase activity at stage 10 . A representative result is shown out of four independent experiments.

\section{Acknowledgement}

The authors would like to thank Drs. David Turner for $\beta$-catenin/ pCS2t, Yoshiki Sasai for Chd/pBS+, Douglas A. Melton for Follistatin/ pBS and Ryoichiro Kageyama for PGV-B-N6-LUC.

\section{References}

ARTINGER, M., BLITZ, I., INOUE, K., TRAN, U. and CHO, K.W. (1997). Interaction of goosecoid and brachyury in Xenopus mesoderm patterning. Mech Dev65: 187-96.

BAE, S., BESSHO, Y., HOJO, M. and KAGEYAMA, R. (2000). The bHLH gene Hes6, an inhibitor of Hes1, promotes neuronal differentiation. Development 127: 2933-43

BLITZ, I.L. and CHO, K.W. (1995). Anterior neurectoderm is progressively induced during gastrulation: the role of the Xenopus homeobox gene orthodenticle. Development 121: 993-1004.

COPELAND, J.W., NASIADKA, A., DIETRICH, B.H. and KRAUSE, H.M. (1996). Patterning of the Drosophila embryo by a homeodomain-deleted Ftz polypeptide. Nature 379: 162-5.

DAVIS, R.L. and TURNER, D.L. (2001). Vertebrate hairy and Enhancer of split related proteins: transcriptional repressors regulating cellular differentiation and embryonic patterning. Oncogene 20: 8342-57.

FISHER, A. and CAUDY, M. (1998). The function of hairy-related bHLH repressor proteins in cell fate decisions. Bioessays 20: 298-306.

GAWANTKA, V., DELIUS, H., HIRSCHFELD, K., BLUMENSTOCK, C. and NIEHRS, C. (1995). Antagonizing the Spemann organizer: role of the homeobox gene Xvent-1. EMBO J14: 6268-79.

GIAGTZOGLOU, N., ALIFRAGIS, P., KOUMBANAKIS, K.A. and DELIDAKIS, C. (2003). Two modes of recruitment of $\mathrm{E}(\mathrm{spl})$ repressors onto target genes. Development 130: 259-70.

GLINKA, A., WU, W., DELIUS, H., MONAGHAN, A.P., BLUMENSTOCK, C. and NIEHRS, C. (1998). Dickkopf- 1 is a member of a new family of secreted proteins and functions in head induction. Nature 391: 357-62.

HARLAND, R. and GERHART, J. (1997). Formation and function of Spemann's organizer. Annu Rev Cell Dev Bio/13: 611-67.

HARLAND, R.M. (1991). In situ hybridization: an improved whole-mount method for Xenopus embryos. Methods Cel/ Bio/36: 685-95.

HAWLEY, S.H., WUNNENBERG-STAPLETON, K., HASHIMOTO, C., LAURENT, M.N., WATABE, T., BLUMBERG, B.W. and CHO, K.W. (1995). Disruption of BMP signals in embryonic Xenopus ectoderm leads to direct neural induction. Genes Dev 9: 2923-35.

HEMMATI-BRIVANLOU, A., KELLY, O.G. and MELTON, D.A. (1994). Follistatin, an antagonist of activin, is expressed in the Spemann organizer and displays direct neuralizing activity. Cel/77: 283-95.

IEMURA, S., YAMAMOTO, T.S., TAKAGI, C., UCHIYAMA, H., NATSUME, T., SHIMASAKI, S., SUGINO, H. and UENO, N. (1998). Direct binding of follistatin to a complex of bone-morphogenetic protein and its receptor inhibits ventral and epidermal cell fates in early Xenopus embryo. ProcNat/Acad Sci USA 95: 933742.

KUROIWA, A., HAFEN, E. and GEHRING, W.J. (1984). Cloning and transcriptional analysis of the segmentation gene fushi tarazu of Drosophila. Ce//37: 825-31.

LEYNS, L., BOUWMEESTER, T., KIM, S.H., PICCOLO, S. and DE ROBERTIS, E.M. (1997). Frzb-1 is a secreted antagonist of Wnt signaling expressed in the Spemann organizer. Cel/88: 747-56.

LÓPEZ, S.L., ROSATO-SIRI, M.V., FRANCO, P.G., PAGANELLI, A.R. and CARRASCO, A.E. (2005). The Notch-target gene hairy2a impedes the involution of notochordal cells by promoting floor plate fates in Xenopus embryos. Development 132: 1035-46.

MCLARREN, K.W., LO, R., GRBAVEC, D., THIRUNAVUKKARASU, K., KARSENTY, G. and STIFANI, S. (2000). The mammalian basic helix loop helix protein HES1 binds to and modulates the transactivating function of the runt-related factor 
Cbfa1. J Biol Chem 275: 530-8.

MESSENGER, N.J., KABITSCHKE, C. ANDREWS, R., GRIMMER, D., NUNEZ MIGUEL, R., BLUNDELL, T.L., SMITH, J.C. and WARDLE, F.C. (2005). Functional specificity of the Xenopus T-domain protein Brachyury is conferred by its ability to interact with Smad1. Dev Cel/8: 599-610.

MIZUSEKI, K., KISHI, M., MATSUI, M., NAKANISHI, S. and SASAI, Y. (1998). Xenopus Zic-related-1 and Sox-2, two factors induced by chordin, have distinct activities in the initiation of neural induction. Development 125: 579-87.

MOOS, M., JR., WANG, S. and KRINKS, M. (1995). Anti-dorsalizing morphogenetic protein is a novel TGF-beta homolog expressed in the Spemann organizer. Development 121: 4293-301.

NEWMAN, C.S., CHIA, F. and KRIEG, P.A. (1997). The XHex homeobox gene is expressed during development of the vascular endothelium: overexpression leads to an increase in vascular endothelial cell number. Mech Dev66: 83-93.

NIEUWKOOP, P.D. and FABER, J. (1967). Normal table of Xenopus laevis (Daudin). A systematical and chronological survey of the development from the fertilized egg till the end of metamorphosis. North-Holland Pub. Co., Amsterdam.

ONICHTCHOUK, D., GAWANTKA, V., DOSCH, R., DELIUS, H., HIRSCHFELD, K., BLUMENSTOCK, C. and NIEHRS, C. (1996). The Xvent-2 homeobox gene is part of the BMP-4 signalling pathway controlling [correction of controling] dorsoventral patterning of Xenopus mesoderm. Development 122: 3045-53.

POOLE, S.J., KAUVAR, L.M., DREES, B. and KORNBERG, T. (1985). The engrailed locus of Drosophila: structural analysis of an embryonic transcript. Cel/40: 37-43.

SANDER, K. and FAESSLER, P.E. (2001). Introducing the Spemann-Mangold organizer: experiments and insights that generated a key concept in developmental biology. Int. J. Dev. Biol. 45: 1-11.

SASAI, Y., LU, B., STEINBEISSER, H., GEISSERT, D., GONT, L.K. and DE ROBERTIS, E.M. (1994). Xenopus chordin: a novel dorsalizing factor activated by organizer-specific homeobox genes. Cel/79: 779-90.

SMITH, J.C., PRICE, B.M., GREEN, J.B., WEIGEL, D. and HERRMANN, B.G.
(1991). Expression of a Xenopus homolog of Brachyury (T) is an immediateearly response to mesoderm induction. Cel/67: 79-87.

SUN, Y., NADAL-VICENS, M., MISONO, S., LIN, M.Z., ZUBIAGA, A., HUA, X., FAN, G. and GREENBERG, M.E. (2001). Neurogenin promotes neurogenesis and inhibits glial differentiation by independent mechanisms. Cel/104: 365-76.

SUZUKI, T., KAWASAKI, H., YU, R.T., UEDA, H. and UMESONO, K. (2001). Segmentation gene product Fushi tarazu is an LXXLL motif-dependent coactivator for orphan receptor FTZ-F1. Proc Natl Acad Sci USA 98: 12403-8.

TAIRA, M., JAMRICH, M., GOOD, P.J. and DAWID, I.B. (1992). The LIM domaincontaining homeo box gene Xlim-1 is expressed specifically in the organizer region of Xenopus gastrula embryos. Genes Dev6: 356-66.

TSUJI, S., CHO, K.W. and HASHIMOTO, C. (2003). Expression pattern of a basic helix-loop-helix transcription factor Xhairy2b during Xenopus laevis development. Dev Genes Evo/213: 407-11.

TSUJI, S. and HASHIMOTO, C. (2005). Choice of either beta-catenin or Groucho/ TLE as a co-factor for Xtcf-3 determines dorsal-ventral cell fate of diencephalon during Xenopus development. Dev Genes Evo/215: 275-84.

WANG, S., KRINKS, M., LIN, K., LUYTEN, F.P. and MOOS, M., JR. (1997). Frzb, a secreted protein expressed in the Spemann organizer, binds and inhibits Wnt8. Cel/88: 757-66.

YAMAGUTI, M., CHO, K.W. and HASHIMOTO, C. (2005). Xenopus hairy2b specifies anterior prechordal mesoderm identity within Spemann's organizer. Dev Dyn 234: 102-13.

YOSHIDA, S., MARIKAWA, Y. and SATOH, N. (1996). Posterior end mark, a novel maternal gene encoding a localized factor in the ascidian embryo. Development 122: $2005-12$.

Received: September 2005

Reviewed by Referees: November 2005

Modified by Authors and Accepted for Publication: January 2006 